\title{
Exposición a plomo en trabajadores de fábricas informales de baterías
}

\author{
Workers' lead exposure in informal batteries factories
}

\author{
Augusto V Ramírez ${ }^{1}$ \\ ${ }^{1}$ Médico del Trabajo. American College of Occupational and Environmental Medicine.
}

\section{Resumen}

Introducción: El problema del trabajo industrial con plomo es universal, sobretodo cuando se trabaja sin criterios de higiene ocupacional. En el Perú la industria minero metalúrgica del plomo mantiene programas de gestión en salud ocupacional de buena calidad, pero no sucede lo mismo en la industria informal del plomo. Objetivos: Investigar el estado de salud de trabajadores de fábricas artesanales e informales de baterías, usando como indicador de exposición nivel de plomo en sangre. Diseño: Estudio de tipo observacional y transversal. Lugar de estudio: Fábricas de baterías del sector informal en el sur del Perú y en el Servicio de Salud Ocupacional Hospital llo, Perú. Participantes: Trabajadores de cuatro fábricas de baterías del sector informal. Intervenciones: La información se obtuvo mediante entrevista directa a 41 trabajadores. El estudio fue realizado entre agosto 1999 y enero 2000. Para medir plomo sanguíneo, se utilizó espectrofotometría de absorción atómica con horno de grafito y para hemoglobina, microhematocrito. Se obtuvo datos en porcentajes, promedios, rangos y desviaciones estándar. Se comparó los resultados de niveles de plomo sanguíneo con variables de salud e higiene ocupacional. Principales medidas de los resultados: Asociación entre plomo sanguíneo y síntomas de saturnismo. Resultados: La edad promedio del grupo fue 35 años (rango: 16 a 52), media de tiempo de trabajo, 6,5 años. El promedio de plomo sanguíneo fue 37,7 ug/dL (rango: 13,5 a 72,3, desviación estándar: 15,7), hemoglobina media 11,2 g/dL (rango: 8,9-13,2, desviación estándar 1,1). Se halló asociación estadística significativa entre plomo sanguíneo elevado y síntomas de saturnismo $(p<0,05)$, trabajo como soldador-fundidor $(p<0,001$ ) y malos hábitos de higiene ocupacional $(p<0,05)$. Conclusiones: La salud del trabajador en este grupo ocupacional se encontró afectada. El valor medio de plomo sanguíneo encontrado fue $37,7 \mathrm{ug} / \mathrm{dL}$ y la hemoglobina hallada en dicha población fue menor de 13,2 g/dL.

Palabras clave: Intoxicación por plomo; exposición ocupacional; salud laboral; industrias; baterías.

\begin{abstract}
Introduction: Work with lead is a universal industrial problem, especially when done without labor hygiene approach. Lead Peruvian's mining and metallurgy maintains good quality programs for management of occupational health, but informal Peruvian industry does not. Objectives: To determine the actual heath status of handicraft and informal batteries factories workers using blood lead levels as exposition indicators. Design: Observational and transversal study. Setting: Ilo, Peru's Occupational Health Service Hospital. Participants: Workers at four handicraft informal batteries factories. Interventions: Information was obtained from 41 workers by direct interview, from August 1999 through January 2000. For blood lead levels determination we used atomic absorption spectrophotometry with graphite oven, and for hemoglobin, microhematocrit. We obtained percentages, averages, ranges, and standard deviations. We compared blood lead levels with health and occupational hygiene variables. Main outcome measures: Blood lead and saturnism symptoms association. Results: Group average age was 35 years (range. 16 to 52 ), work time average, 6,5 years. Blood lead level: 37,7 [ $\pm 17,7] \mathrm{ug} / \mathrm{dL}$ (range: 13.5 to 72,3 ). Mean hemoglobin: $11,2[ \pm 1,1] \mathrm{g} / \mathrm{dL}$ (range: 8,9 to 13,2 ). We found significant statistical association between blood lead level $>30 \mathrm{ug} / \mathrm{dL}$ and occupational lead poisoning, work as welder-caster and worse habits of occupational hygiene. Conclusions: Worker's health is affected in this occupational group. Blood lead levels exceeded permissible levels for exposed workers and they showed anemia.
\end{abstract}

Key words: Lead poisoning; occupational exposure; industry; batteries; occupational health.

\section{INTRODUCCIÓN}

La contaminación de los lugares de trabajo es un problema actual de salud ocupacional, principalmente por la fundición primaria o secundaria de metales pesados en enormes hornos pirometalúrgicos. Existen otras causas que pasan desapercibidas, como la contaminación producida por el mosaico de pequeñas industrias clandestinas insertas en las ciudades. La más conspicua de estas actividades es la fabricación artesanal de acumuladores eléctricos a partir del reciclaje de baterías y la consiguiente liberación de vapores de plomo, que constituye un problema ocupacional y ambiental oculto, propio de los sectores de 'economía sumergida', del que solo ocasionalmente se escucha voces de alerta, por lo general periodísticas ${ }^{(1-4)}$.

Esta industria se inicia con la compra callejera de las baterías en desuso, las que son desguazadas para recuperar el plástico, el ácido y todas las piezas de plomo (bornes, placas positivas y negativas, puentes y rejillas), que luego son fundidas en ambientes precarios, como cocinas domésticas ventiladas solo con pequeños ductos, pues por su carácter clandestino se las mantiene cerradas. El plomo fundido se moldea para fabricar artesanalmente las piezas de 'nuevas' baterías y para otros usos, como fabricación de alambre para 'plomos' a usar en fusibles eléctricos caseros, etc. ${ }^{(5)}$.

En exposición ocupacional, la toxicología del plomo es conocida: sus vapores y humos que llegan al pulmón son absorbi- dos en un $50 \%$. La sangre distribuye este metal a todo el organismo, donde puede lesionar órganos blandos, como el sistema nervioso central y periférico. Pero el daño más temprano y ostensible lo causa en la sangre, al interferir la síntesis de globulina en el hematíe, bloqueando la fijación del hierro a la protoporfirina IX, en la formación del núcleo hem. Esto condiciona que la protoporfirina IX se una al zinc y forme zinc protoporfirina (ZPP), que aumenta su concentración en el hematíe. El resultado final de este daño es la anemia normocítica e hipocrómica, con aumento secundario del hierro sérico. Finalmente, el plomo es excretado en $75 \%$ por los riñones, a los que también puede lesionar. El plomo es un neurotóxico muy peligroso para el 
desarrollo embrionario del cerebro y de allí el riesgo de embarazo en mujeres de edad fértil expuestas ${ }^{(6,7)}$.

Para el monitoreo biológico en exposición ocupacional, se usa como indicador el plomo en sangre $(\mathrm{Pb}-\mathrm{S})$, por reflejar con fidelidad sus concentraciones medias en los compartimentos de recambio. Sin embargo, existen otros indicadores biológicos de exposición (BEIs): en sangre, hemoglobina, en hematíes, zinc-protoporfirina y actividad de la dehidratasa del ácido $\Delta$ amino-levulínico (ALA $\Delta$ - D); en orina, la excreción de ácido $\Delta$ amino-levulínico (ALA $\Delta$ ), etc. En la práctica diaria, el indicador más usado es el plomo sanguíneo y los otros quedan reservados para solucionar dificultad diagnóstica, diagnóstico diferencial o valoración de daño bioquímico ${ }^{(8,9)}$.

Con estos antecedentes, nos propusimos determinar el estado de salud de una población de trabajadores expuestos a plomo inorgánico, en fábricas informales de reciclaje de baterías, localizadas en asentamientos humanos periféricos del sur peruano. Ello nos permitiría un acercamiento al estado actual de salud de este grupo ocupacional que, al ser informal, no es protegido, trabaja en condiciones inseguras y se contamina; hecho agravado, porque entre estos trabajadores se encuentran mujeres y adolescentes, cuyo organismo es mucho más susceptible al tóxico.

\section{MÉTODOS}

Se realizó un estudio de tipo observacional y transversal ${ }^{(10)}$. La población estudiada estuvo constituida por 41 trabajadores de cuatro fábricas artesanales de reciclado de baterías, ubicadas en el sur del Perú: Ilo y Tacna. Usamos como indicadores de exposición niveles de plomo sanguíneo ( $\mathrm{Pb}-\mathrm{S}$ ) y niveles de hemoglobina en sangre. La información de hábitos higiénico-laborales se obtuvo mediante entrevista directa a trabajadores. El estudio se realizó entre agosto de 1999 y enero de 2000, con participación voluntaria de los trabajadores, previo su consentimiento informado y aceptación voluntaria y respetándose lo recomendado en la Declaración de Helsinki ${ }^{(11)}$.

Se consideró los siguientes grupos:

- Trabajadores expuestos directos: los que manipulan plomo en el proceso de reciclaje (fundidores, soldadores, torneros, rejilladores y ensambladores) y

- Trabajadores expuestos indirectos: aquellos que laboran como 'personal de apoyo' en oficina y ayudantes o aprendices en el reciclaje de las baterías, grupo que no se expone directamente al tóxico, pero que podría estar contaminado por laborar en lugares colindantes al área de fundición de este metal.

Se incluyó a todo trabajador que se encontrase laborando un tiempo mínimo de un año en las fábricas, al momento de la evaluación. Una de las limitaciones metodológicas del presente estudio fue la magnitud del universo, pues al ser informales solo ubicamos por investigación propia y directa estas fábricas; la otra, más importante, fue el acto médico, que en sí no reunió condiciones óptimas de higiene ocupacional, pues para la entrevista, examen y toma de muestra elegimos un lugar aislado, pero dentro del área laboral.

Con anamnesis y examen clínico ocupacional exploramos síntomas y signos aceptados en toxicología como propios de exposición a plomo (cólico saturnino, paresias, parestesias o línea gingival) y otros que, sin ser clásicos, siempre los encontramos asociados a esta exposición ${ }^{(6,7)}$.

Para la medición de $\mathrm{Pb}$-S y hemoglobina, se tomó $5 \mathrm{~mL}$ de sangre venosa en un tubo Vacutainer ${ }^{\circledR}$, específico para muestra de $\mathrm{Pb}-\mathrm{S}$, el mismo que se encontraba libre de metales pesados, y con ácido etilen diamino tetracético (EDTA), como anticoagulante. La muestra permaneció refrigerada entre 18 y 24 horas, mientras se la transportaba vía terrestre, para el análisis de plomo en un espectrofotómetro de absorción atómica, Perkin Elmer 603, con horno de grafito, aparato calibrado periódicamente para validación de su exactitud y precisión, antes de cada corrida. Se realizó la medición de hemoglobina con el método del microhematocrito.

Se utilizó porcentajes, medias aritméticas y estándares de desviación de los valores de $\mathrm{Pb}$-S y hemoglobina para evaluar estadísticamente los resultados; los cuales fueron comparados con valores medios de $\mathrm{Pb}-\mathrm{S}$ de variables de salud e higiene ocupacional, estableciéndose el valor de ' $t$ ' por la prueba $t$ de Fisher, y el significado de las diferencias mediante cálculo de la probabilidad 'p', considerándose estadísticamente significativos aquellos valores de $\mathrm{p}<0,05$. Para el procesamiento de datos se utilizó el programa Excel® y los resultados se presenta en tablas. Como valor del indicador biológico de exposición (BEI) de $\mathrm{Pb}-\mathrm{S}$, adoptamos el de la American Conference of Governmental Industrial Hygienist (ACGIH) que, en el año 2007, estableció valores para trabajador varón adulto expuesto como menor a $30 \mathrm{ug} / \mathrm{dL}$ y menor a $10 \mathrm{ug} / \mathrm{dL}$ para la mujer en edad fértil expuesta. Para los menores de 18 años de edad, seguimos las pautas del Center for Disease Control (CDCEEUU), que establece el límite como igual o menor de $10 \mathrm{ug} / \mathrm{dL}^{(12,13)}$. Nuestra norma peruana Guía de Práctica Clínica para el Manejo de Pacientes con Intoxicación por Plomo establece un valor menor a $40 \mathrm{ug} / \mathrm{dL}$ para el trabajador expuesto ${ }^{(14)}$.

\section{RESULTADOS}

Las características generales de la población estudiada fueron: de los 41 trabajadores participantes en el estudio, 34 (83\%) fueron varones y $7(17 \%)$ mujeres. Del grupo masculino, 9 (22\% del total y $26 \%$ del grupo) fueron adolescentes. La edad promedio del universo fue 43 años (rango 16 a 50) (Tabla 1).

La Tabla 2 presenta los resultados generales referidos a plomo sanguíneo y hemoglobina: el valor medio de plomo sanguíneo hallado fue $37,7 \pm 15,7 \mathrm{ug} / \mathrm{dL}$, el mismo que se encuentra por encima de los límites máximos recomendados para exposición ocupacional ${ }^{(12,13)}$. El valor medio a de hemoglobina hallada fue $11,22 \pm 1,1$ $\mathrm{g} \%$, valor no considerado normal para el peruano del llano ${ }^{(15)}$.

En cuanto a lo hallado en sintomatología, la dividimos en:

- Síntomas relacionados al trabajo con plomo: destacan los neurológicos (26\%) y el cólico sin diarrea (29\%); y

- Síntomas asociados al método con el cual se procesa el plomo y no necesariamente asociados directamente al metal: irritación ocular (76\%), cefalea global (63\%) y dolor de garganta (41\%) (Tabla 3).

Específicamente, en el campo neurológico hallamos que $24 \%$ presentaba parestesias, hormigueo o adormecimiento en miembros y un trabajador fundidor-soldador

Tabla 1. Características de la población expuesta a plomo $(n=41)$.

\begin{tabular}{lrcc}
\hline $\begin{array}{c}\text { Ocupación del } \\
\text { trabajador }\end{array}$ & $\mathrm{N}^{0}$ & $\begin{array}{c}\text { Media y rango } \\
\text { de edad (años) }\end{array}$ & $\begin{array}{c}\text { Media de tiempo } \\
\text { de trabajo (años) }\end{array}$ \\
\hline Fundidor & 4 & $41(25-51)$ & 10 \\
Soldador & 5 & $44(33-52)$ & 8 \\
Ensamblador & 5 & $37(28-45)$ & 5 \\
Tornero & 6 & $39(30-48)$ & 9 \\
Rejillador & 5 & $37(30-52)$ & 8 \\
Total trabajadores directos & $\mathbf{2 5}$ & $40(25-52)$ & 8 \\
Aprendiz & 7 & $17(16-17,8)$ & 2 \\
Total trabajadores varones & 32 & $35(16-52)$ & 7 \\
Apoyo oficina & 9 & $35(29-38)$ & 4 \\
Total universo & 41 & $35(16-52)$ & 6,5 \\
\hline
\end{tabular}


Tabla 2. Niveles de plomo y hemoglobina séricos hallados en la población estudiada ( $n=41)$.

\begin{tabular}{|c|c|c|c|c|c|}
\hline Ocupación del trabajador & $\mathrm{N}^{0}$ & $\begin{array}{c}\text { Media Pb-S* ug/dL } \\
\left( \pm \mathrm{DE}^{\ddagger}\right)\end{array}$ & Rango $\mathrm{Pb}-\mathrm{S}^{*}$ ug/dL & $\begin{array}{l}\text { Media } \mathrm{Hb}^{\dagger} \mathrm{g} \% \\
\left( \pm \mathrm{DE}^{\ddagger} \mathrm{Hb}\right)\end{array}$ & Rango $\mathrm{Hb}^{\dagger} \mathrm{g} \%$ \\
\hline \multicolumn{6}{|l|}{ Adultos varones: } \\
\hline Fundidor & 4 & $61,9( \pm 9,0)$ & $50,6-72,3$ & $9,7( \pm 0,81)$ & $8,90-10,8$ \\
\hline Soldador & 5 & $60,14( \pm 2,8)$ & $56,9-62,7$ & $11,4( \pm 0,7)$ & $9,60-11,2$ \\
\hline Ensamblador & 5 & $42,74( \pm 2,5)$ & $40,0-45,6$ & $12,1( \pm 1,2)$ & $10,0-13,1$ \\
\hline Tornero & 6 & $30,75( \pm 4,5)$ & $25,9-36,4$ & $11,5( \pm 0,7)$ & $10,6-12,3$ \\
\hline Rejillador & 5 & $40,46( \pm 7,5)$ & $33,1-50,0$ & $11,3( \pm 1,1)$ & $10,2-13,0$ \\
\hline Total Trabajadores directos & 25 & $45,9( \pm 13,2)$ & $25,9-72,3$ & $11,1( \pm 1,1)$ & $8,90-13,1$ \\
\hline \multicolumn{6}{|l|}{ Adolescentes: } \\
\hline Aprendiz & 7 & $24,0( \pm 9,2)$ & $14,9-35,4$ & $11,0( \pm 1,1)$ & $9,20-12,6$ \\
\hline Total Trabajadores varones & 32 & $41,14( \pm 15,2)$ & $14,9-72,3$ & $11,07( \pm 1,2)$ & $8,90-13,1$ \\
\hline \multicolumn{6}{|l|}{ Mujeres: } \\
\hline Apoyo oficina & 9 & $25,6( \pm 10,3)$ & $13,5-38,2$ & $11,64( \pm 1,0)$ & $10,0-13,2$ \\
\hline Total Universo & 41 & $37,7( \pm 15,7)$ & $13,5-72,3$ & $11,22( \pm 1,1)$ & $8,90-13,2$ \\
\hline
\end{tabular}

"Plomo sanguíneo. ${ }^{\dagger}$ Hemoglobina. ${ }^{\ddagger}$ Desviación estándar de la media.

Tabla 3. Hallazgos clínicos (signos y síntomas) encontrados en la población estudiada $(n=41)$.

\begin{tabular}{lrr}
\hline \multicolumn{1}{c}{ Síntoma / Signo } & $\mathrm{n}$ & $\%$ \\
\hline 1. Asociados a trabajo con plomo: & & \\
Sensación de sabor metálico & 23 & 56 \\
Palidez marcada de piel y mucosas & 19 & 46 \\
Dolor cólico sin diarrea, ocasional & 12 & 29 \\
Parestesias miembros superiores & 10 & 24 \\
Línea gingival & 3 & 7 \\
Paresia cubital & 1 & 2 \\
2. Asociados al método de trabajo: & & \\
Cefalea global & 26 & 63 \\
Irritación ocular & 31 & 76 \\
Dolor de garganta & 17 & 41 \\
Visión borrosa al final de la jornada laboral & 14 & 34 \\
\hline
\end{tabular}

(2\%), tuvo paresia cubital, con $\mathrm{Pb}-\mathrm{S}$ de 72,3 ug/dL.

Los hallazgos de importancia en condiciones de exposición se muestra en la Tabla 4:

- Del grupo de expuestos directos: 2 soldadores $(8 \%)$ y un fundidor $(4 \%)$ habían trabajado en actividades con exposición a plomo en empresas informales de la misma actividad en Lima.

- Del grupo total, 3 (7\%) habían tenido un examen de $\mathrm{Pb}-\mathrm{S}$ y en 2 de ellos (5\%) fue mayor a $50 \mathrm{ug} / \mathrm{dL}$, pero ninguno consultó a un médico y siguieron trabajando en las mismas condiciones.

En cuanto a trabajo y medidas de higiene industrial del grupo total, encontramos que solo 12 trabajadores (29\%) conocían y ejecutaban hábitos de higiene; 22 (54\%) tomaban sus alimentos en el área de labor y $6(15 \%)$ habían recibido algún tipo de capacitación para trabajo con plomo. De estos últimos, 3 (12\% del grupo de mayor exposición y 7\% del universo) se cambiaban la ropa de trabajo por ropa de calle al término de su labor y solo un trabajador $(2,4 \%)$ usaba respirador adecuado al riesgo y le cambiaba filtros periódicamente.

Se halló significancia estadística en la asociación $\mathrm{Pb}-\mathrm{S}>30 \mathrm{ug} / \mathrm{dL}$ y ocupación de fundidor-soldador $(\mathrm{p}<0,001), \mathrm{Pb}-\mathrm{S}>$ $30 \mathrm{ug} / \mathrm{dL}$ y síntomas atribuibles a plomo (cólicos, neurológicos, línea gingival) y $\mathrm{Pb}-\mathrm{S}>30 \mathrm{ug} / \mathrm{dL}$ y malos hábitos de higiene y seguridad en el trabajo $(p<0,05)$.

\section{DISCUSIÓN}

El plomo es uno de los metales pesados más ubicuos y dañinos. En salud laboral, se encuentra muy bien establecida su acción tóxica ${ }^{(7,8,15,16)}$, hecho agravado por la domiciliarización de los riesgos ocupacionales ${ }^{(17)}$. El plomo reconoce al reciclaje y fabricación secundaria de baterías como una de las actividades que causa mayor contaminación laboral y ambiental $(5,18,19)$. En varias ciudades peruanas existe un sector de trabajadores que labora en esta actividad y lo hace en precarias condiciones de higiene y seguridad ocupacional ${ }^{(20-23)}$ y sabemos que la exposición ocupacional al plomo sin protección adecuada determina intoxicación saturnina de diagnóstico arduo para el médico en general, y de ahí su importancia en salud pública $(7,15,19,24,25)$.

Nuestro estudio verifica que lo precario del proceso de fundido de plomo en el sector industrial informal es fuente importante de exposición al tóxico, por no contar estos trabajadores con medidas colectivas de protección. En estas condiciones de trabajo, el riesgo de intoxicación ocupacional por plomo es alto y afecta no solo a los trabajadores -que a veces son los mismos dueños de la empresa-, sino que podría también afectar a otros miembros de sus familias.

El valor medio de plomo sanguíneo $(37,7$ $\pm 15,7 \mathrm{ug} / \mathrm{dL}$ ) encontrado está por encima de los límites máximos recomendados para exposición ocupacional ${ }^{(12,13)}$, pero $3 \mathrm{ug} / \mathrm{dL}$ por debajo del valor dado en la norma peruana como máximo permisible ${ }^{(14)}$. El valor medio de hemoglobina $(11,22 \pm 1,1 \mathrm{~g} \%)$ se encontró por debajo de lo considerado normal para el peruano del llano ${ }^{(16)} \mathrm{y}$, en general, ambos valores medios de plomo y hemoglobina encontrados en el presente estudio, concuerdan con los hallados en otros estudios peruanos ${ }^{(16,20-23)}$ y extranjeros ${ }^{(26-29)}$, en poblaciones de exposición similar.

El cuadro clínico que presentaron los trabajadores en nuestro estudio estaba condicionado por la ausencia de medidas individuales y colectivas de higiene y seguridad (mala ventilación del área laboral, control nulo del proceso, no usar o usar respiradores inadecuados para el riesgo, no usar ropa de protección personal en el trabajo) y malos hábitos de higiene, como tomar los alimentos en el lugar de trabajo, hechos que se agravaban por la ausencia de programas de capacitación y prevención-promoción de la salud en el trabajo, que hagan conocer a los trabajadores y a los dueños de las fábricas

Tabla 4. Hábitos higiénico-laborales en la población estudiada expuesta a plomo ( $n=41)$

\begin{tabular}{|c|c|c|}
\hline Tipo & $\begin{array}{c}\text { Siempre } \\
\mathrm{n}(\%)\end{array}$ & $\begin{array}{c}\text { Nunca } \\
\mathrm{n}(\%)\end{array}$ \\
\hline \multicolumn{3}{|l|}{$\begin{array}{l}\text { 1. Uso de equipo de protección personal } \\
\text { Ropa de trabajo: }\end{array}$} \\
\hline \multicolumn{3}{|l|}{ Incompleta: } \\
\hline Mameluco cuerpo entero & $6(15)$ & $35(85)$ \\
\hline Zapatos de seguridad & $4(10)$ & $37(90)$ \\
\hline Respirador con filtro para trabajo con plomo & $1(2,5)$ & $40(97,5)$ \\
\hline Cambia filtro de acuerdo a la norma de higiene & $1(2,5)$ & $40(97,5)$ \\
\hline Careta de soldar (soldadores/ fundidores $\mathrm{n}: 9)^{\circ}$ & $4(44)$ & $5(56)$ \\
\hline Cambia ropa trabajo por ropa calle & $3(7)^{\prime}$ & $38(93)$ \\
\hline \multicolumn{3}{|l|}{ 2. Normas de higiene: } \\
\hline Baño diario al final de las jornada laboral & $12(29)$ & $29(71)$ \\
\hline Lavado manos antes de tomar alimentos y agua & $12(29)$ & $29(71)$ \\
\hline Toma alimentos dentro del área de trabajo & $22(54)$ & $19(46)$ \\
\hline \multicolumn{3}{|l|}{ 3. Antecedentes: } \\
\hline Trabajo anterior expuesto a plomo & $3(7)$ & $38(93)$ \\
\hline Examen medico general de ingreso & $6(15)$ & $35(85)$ \\
\hline Examen medico ocupacional anual & $3(7)$ & $38(93)$ \\
\hline Examen de plomo sanguíneo el año anterior & $3(7)$ & $38(93)$ \\
\hline Examen $\mathrm{Pb}$-s recuerda su resultado & $2(5)$ & 39 (95) \\
\hline Si su $\mathrm{Pb}-\mathrm{S}^{\dagger}$ fue $>30 \mathrm{ug} / \mathrm{dL}$, tuvo consulta médica? & 0 & $100 \%$ \\
\hline Habito de fumar: & $13(41)$ & $19(59)$ \\
\hline \multicolumn{3}{|l|}{ 4. Lugar de residencia: } \\
\hline Dentro del perímetro urbano de la ciudad & $17(41)$ & \\
\hline Barrio periférico a la ciudad & $24(59)$ & \\
\hline 5. Capacitación laboral para trabajar con plomo & $6(15)^{\prime}$ & $35(85)$ \\
\hline
\end{tabular}

"Careta de soldar de uso colectivo: una por fábrica. ${ }^{\dagger}$ Plomo sanguíneo. 
los efectos que sobre la salud produce la exposición a este metal.

En conclusión, encontramos niveles elevados de plomo sanguíneo, síntomas asociados a intoxicación por ese metal y malas condiciones higiénico-laborales en este grupo de trabajadores informales de baterías en el sur de nuestro país.

\section{AGRADECIMIENTOS}

El autor agradece a los trabajadores y directivos de las empresas de fabricación artesanal de baterías, por su participación en esta investigación.

A la Dra. Lucía Medina R, salubrista del entonces Centro de Salud John F. Kennedy de Ilo, por el apoyo en el trabajo de campo, a los internos del Hospital Ilo de la Universidad Nacional San Agustín de Arequipa, por su colaboración en las diferentes fases del estudio, y a la Srta. Maribel Arias Ucero, por la coordinación y apoyo en la localización de las fábricas y el mecanografiado del manuscrito.

\section{REFERENCIAS BIBLIOGRÁFICAS}

1. Brack EA. Perú y ambiente, uno de los desafíos del siglo XXI. Lima: Asociación Nacional de Centros, IV Conades-PNUD; 2000.

2. Weitzenfeld H. Contaminación atmosférica y salud en América Latina. Bol Oficina Sanit Panam. 1992;112:97-109.

3. Gamero EC. Humo y fábricas nos ahogan. Diario 0J0. Lima, Perú. Edición 5 de junio de 2001. p. 14.

4. Diario La República. Marchan contra contaminación por plomo. Lima, Perú. Edición 13 de junio, 2007.

5. Florez W, Canahua H, Mamani E. Reciclaje de baterías usadas de plomo-ácido para su aplicación en la enseñanza de química en la Universidad Nacional Jorge Basadre Grohman, Tacna. 3er Congreso Peruano de Materiales. Arequipa-Perú, 2004.

6. Ellemhorn MJ. Medical Toxicology. Penn, USA: William \& Wilkins; 1997.

7. Ramírez AV. El cuadro clínico de la intoxicación ocupacional por plomo. An Fac Med. 2005;66(1):5770.
8. Zenz C, ed. Occupational Medicine. 3rd Edition. St. Louis, USA: Ed Mosby - Year Book Inc.; 1994.

9. Levy BS, Wegman DH, eds. Occupational Health. Boston: Little, Brown and Co.; 1988.

10. Reynaga 0J. Epidemiología IV: diseños de investigación para la comprobación de hipótesis epidemiológicas. Estudios transversales y estudios retrospectivos. México, DF: Centro Panamericano de Ecología Humana y Salud, Organización Panamericana de la Salud; 1985.

11. Asociación Médica Mundial. Declaración de Helsinki. Principios éticos aplicables a las investigaciones médicas que involucran seres humanos. 18 Asamblea General, Helsinki, junio 1964. Ultima Revisión: 52 Asamblea General, Edimburgo, Escocia, octubre 2000.

12. ACGIH, TLVs, BEls. Threshold limits values for chemical substances and physical agents. Biological exposure indices. Cincinnati: ACGIH; 2007.

13. CDC. Preventing lead poisoning in young children. Atlanta, GA: US Department of Health and Human Service; 1991. p. 57-9.

14. Ministerio de Salud del Perú. Guía de práctica clínica para el manejo de pacientes con intoxicación por plomo. Lima: Minsa; 2007.

15. Ramírez AV, Cam PJ, Medina JM. Plomo sanguíneo en los habitantes de cuatro localidades peruanas. Rev Panam Salud Pública. 1997;1(5):344-8.

16. Ramírez AV, Tapia G. Saturnismo en la industria metalúrgica de la altura. Bol Soc Peruana Medicina Interna. 1997;10:153.

17. Pastore J. A agonia do Emprego. Sao Paulo: Ed. Renacemento; 1998.

18. Instituto Nacional de Estadística e Informática, Perú. Informe técnico. Estadísticas Ambientales. Dirección General de Salud Ambiental - DIGESA. Lima: Minsa; 2000.

19. Coya B, MarañónE, Sastre H. Eco toxicidad de las escorias procedentes del reciclado de plomo de baterías agotadas. Revista Técnica Residuos. 1999;9(49):125-83.

20. Anaya R. Saturnismo en el Perú. CICOTOX [sitio en Internet]. Lima: Facultad de Farmacia y Bioquímica, UNMSM. [citado 30 marzo de 2007]. Disponible en: http://www.unmsm.edu.pe/farmacia

21. Machuca SN. Determinación comparativa de plomo en personas expuestas ocupacional e individualmente en jirones Puno y Antonio Raymondi. Tesis para optar el título de Bachiller.
Lima, Perú: UNMSM; 1998.

22. Hoces PN. Riesgo de toxicidad por plomo en trabajadores de industrias de Lima controladas en el Instituto Nacional Salud Ocupacional periodo 89-90. Tesis para optar el título de Bachiller. Lima, Perú: UNMSM; 1999

23. Ubillus $\downarrow$. Estudio sobre la presencia de plomo en el medio ambiente de Talara año 2003. Tesis para optar el título de Bachiller. Lima, Perú: UNMSM; 2003.

24. Barzola AG. Enfermedades subyacentes más frecuentes asociadas a neumonía resistente en Pediatría. Tesis para optar el título de Bachiller. Lima, Perú: UNMSM; 2002.

25. La Dou J, Jackson RT, Howard JJ. Salud ambiental. En: La Dou J. Medicina Laboral y Ambiental. $2^{\mathrm{a}}$ Ed. México: Ed. Manual Moderno; 1999.

26. Aguilar G, Piacitelli GM, Juárez CA, Vázquez JH, Hu H, Hernández M. Exposición ocupacional a plomo inorgánico en la ciudad de México. Salud Pública Méx. 1999;41:42-54.

27. Nogueira DP. Taxa de chumbo em amostra de voluntários "não expostos" habitantes da Grande São Paulo. Rev Bras Saúde Ocupacional. 1980;30:36-8.

28. Uribe C, Gutiérrez M, Patiño N, Uribe M, Martínez 0 , Grool $\mathrm{H}$ y col. Prevalencia de intoxicación por plomo en trabajadores de fábricas de baterías y estudio comparativo entre una prueba de tamizaje y una prueba "de oro": (ZPP-HF) vs. (PBS-EAA). Bogotá: Instituto de los Seguros Sociales; 1996.

29. Cárdenas B0, Varona UM, Núñez TS, Ortiz VJ, Peña PG. Correlación de protoporfirina zinc y plomo en sangre en trabajadores de fábricas de baterías. Bogotá, Colombia. Salud Pública Méx. 2001;43:203-10.

Manuscrito recibido el 23 de mayo de 2008 y aceptado para publicación el 20 de junio de 2008.

\section{Correspondencia:}

Dr. Augusto V. Ramirez, MD.

Los Naranjos 139 B. El lingenio.

POB 110. Serpost.

Cajamarca, Perú.

Correo-e: augustovram@yahoo.es 